\title{
A Review of Psi Activity in the DNA
}

\author{
Bradley Y. Bartholomew \\ Independent Researcher, France
}

Copyright $\bigcirc 2017$ by authors, all rights reserved. Authors agree that this article remains permanently open access under the terms of the Creative Commons Attribution License 4.0 International License

\begin{abstract}
This paper presents a general summary of psi experiments conducted with the DNA in the latter decades of the $20^{\text {th }}$ Century particularly at the HeartMath Institute (HMI) in Boulder Creek, CA, by Dr. Glen Rein, relating to the ability of "healers" to affect the conformation of the DNA molecule simply by concentrating their mental and emotional powers of the brain and heart. In particular it was found that the intentional states of healers could change the molecular structure of water which in turn could alter the conformation of the DNA. Further to these findings about the relationship between water and DNA, this paper discusses a journal article by a team led by 2008 Nobel Prize for Medicine recipient Dr. Luc Montagnier, which describes an electromagnetic field in the DNA that is capable of being projected into water and which contains all the information of its sequence of bases in memory, and is capable of reconstructing itself into the identical chemical molecule; provided the ambient electromagnetic environment in the water is maintained at a frequency of not less than $7 \mathrm{~Hz}$ which is in the same frequency window as Alpha rhythm brainwaves and the natural Schumann resonance of the Earth. This paper goes on to discuss new research in optogenetics where human beings have been able to modify gene expression with their Alpha rhythm and $\mathrm{Mu}$ rhythm brainwaves in the same frequency window as the Schumann resonance, simply by adopting different mental states, and takes note of the fact that these same Alpha and mu rhythms are the main conduit frequency for Brain Computer Interfaces (BCIs). The paper presents a general summary of earlier research relating to psi phenomena resulting from interference of brainwaves with the Schumann resonance, and proposes a novel explanation for the recently discovered "mirror neurons" phenomena where the Mu rhythm brainwaves emanating from the motor cortex are able to interfere constructively or destructively with the Schumann resonance of same frequency and thus bring about mental telepathic as well as psychokinesis happenings.
\end{abstract}

Keywords EMR, Biophotons, Aleister Crowley, Psychic, Shaman, Fritz-Albert Popp, DNA Phantom Effect

\section{Introduction}

Over the past 40 years there has been a great deal of research relating to psi activity in the DNA. Many researchers found electromagnetic radiation (EMR) at varying frequencies being emitted from the DNA. Other researchers have reported the ability of healers to make changes in the DNA even at great distances. Other researchers have pointed out the similar frequencies between our alpha brain waves and the natural resonance of the Earth's atmosphere, the so-called Schumann resonance. Two recent research papers, one in 2010 and the second in 2015 , have now strongly suggested the link between all three of these diverse phenomena, that is to say it is now clear that the EMR from the DNA and the alpha brain waves and the Schumann resonance are all operating in the same frequency window; thus providing a plausible explanation for all psi activity in humans, as well as the ability of plants and the lesser creatures to communicate with each other without having the benefit of the linguistic means of communication that we humans enjoy. Before dealing with the two groundbreaking research papers I must first give a brief summary of prior research in this area.

\section{Early Psi Research in DNA}

Much of the pioneering research into psi effects in the DNA took place in the 1980s and 1990s at the HeartMath Institute (HMI) in Boulder Creek, CA. In 1996 Dr. Glen Rein wrote a landmark paper "Effect of Conscious Intention on DNA" where he summarized all the known mind-matter phenomena pertaining to the DNA [1]. He made reference in his introduction to several studies concerning the effects of focused intention on both physical and biological systems, effects which are commonly labeled under the heading of Psychokinesis.[2, 3]

Rein noted that there were many parallel studies and reports from various types of healers who can produce biological effects simply by willing the patient to be healed [4], as well as Chi-Gong practitioners who claim successes in this area. However he states that in the main these studies are merely phenomenological, and content themselves with 
simply demonstrating "an energetic communication" between the healer and the patient. They make no attempt at explaining the precise biological processes that are occurred which bring about a successful result; that being of course because they didn't actually know the true nature of this power that they have. They were no doubt clear that the power was coming from their own subconscious mind and that it was conjured up when they concentrated their mind on the task at hand.

Some practitioners were however conducting experiments in an effort to pinpoint the source of this "energetic communication", for instance whether a healer could direct his/her intention to protect bacterial cells in culture from the effects of an antibiotic.[5] Rein himself was investigating whether his healer, Leonard Laskow, could inhibit the growth of bacterial cells in culture without using antibiotics. In those days two different healing states of consciousness were distinguished, qualitative healing which was simply to surrender one's will to the will of God and goal-directed which actually involved the focused intention of the healer, and it was found that even such a generalized difference in the mind of the healer could have biological effects on the growth of yeast and seeds.[6]

Rein set out to study the effects of intentionality on DNA synthesis in cultured tumor cells.[7] He was looking for the biological effects of different images, thoughts and intentions, but now he is specifically directing his enquiry at the DNA itself. The tumor cells were in culture and could be precisely monitored. His healer, Laskow, engendered different states of consciousness and focused his intention on three petri dishes, and in an adjacent room aliquots of the same population of cells were presented to a non-healer who was actually reading a book and was trying not to think about the cells at all. Laskow directed five different types of intentional thoughts at the culture and it was found that he achieved a $39 \%$ inhibition when he tried to intentionally will the cells to return to the natural order and harmony of their normal growth rate, and a $21 \%$ inhibition when he simply allowed God's will to manifest. Simply showing the cells unconditional love had no effect at all. Laskow was also able to achieve biological effects through mental imagery. An image in his mind of visualizing only three cells remaining in the petri dish induced an $18 \%$ inhibition in DNA synthesis, and when he generated an image in his mind of many more cells remaining, the growth of the tumor cells increased by $15 \%$.

The effect of intentionality on the conformation of the DNA helix was also studied by Rein and other researchers at the HeartMath Institute. Studying ECG patterns they found that individuals in the state of love have a coherent EM field radiating from their hearts which can maintain homeostasis and promote health in general. It was postulated that the DNA acted as an antennae for the energy fields of the heart and could mediate physiological changes. Interestingly, they saw the DNA picking up the coherent bio-field being transmitted from the heart and dispersing the information to local biochemical reactions inside the cell.

At the time Rein was writing his paper, psychoneuroimmunologists did not consider mind-body effects to be mediated by endogenous EM fields, however he asserts that EEG and ECG measurements have found endogenous bio-fields generated "electrically excitable cells" in the brain and heart. He also refers generally to the findings that the DNA is capable of emitting coherent biophotons, that the DNA molecule actually oscillates coherently, that the DNA is sensitive to externally applied coherent EM fields, that the DNA responds to ordinary EM fields, and that quantum fields enhance DNA synthesis.

Rein lists a number of experiments indicating bio-fields from the heart affect the DNA.[7-11] These experiments were carried out by the Quantum Biology Research Lab and at HMI. In each of these experiments they used isolated DNA in an aqueous solution. A pooled sample of DNA from the placenta of many individuals was placed in a beaker in front of an individual intending to change the DNA. They measured the winding and unwinding of the two strands which make up the DNA helix. The same healer, Leonard Laskow, used a combination of directed intention, unconditional love and specific imagery of the DNA molecule and a direct energetic link was found causing the DNA molecule to wind or unwind. Rein speculated that his healer Laskow could have produced even greater effects if they had used his own DNA in the sample instead of pooled DNA from diverse individuals. These experiments were confirmed at the HMI this time using ordinary subjects (not gifted healers) and this time looking for a correlation between ECG coherence and the changes in the winding or unwinding of the DNA double helix. The more ECG coherence the better able were the subjects to resonate and change the DNA.

Lew Childre, a particularly gifted individual with strong ECG coherence and focused heart intention was able to bring about specific results in three different samples of DNA placed in front of him. Indeed Childre also demonstrated that he could bring about the same changes in the DNA at a distance of 0.5 miles. [9] In these experiments he was notified by a mobile phone when the DNA sample was placed on the laboratory bench and Childre was then able to focus a specific intention to cause the DNA to wind or unwind. Rein states "These results also demonstrate that although coherent heart energy has an electromagnetic component which can act locally within the body, it also has a non-electromagnetic component which is able to carry specific information over long distances. Of course it is well known that healers can project their energy and heal someone thousands of miles away." An attempt was actually made with a healer Valerie Sadyrin who was working with Rein in his California lab [11], to try this experiment when he returned to Moscow. The experiments were conducted twice with a week break in between. The first time the intended changes were made in the DNA sample but not in the second.

Many experiments were conducted by Rein and others and 
there were a lot of variables and mixed successes. According to Rein, only healers capable of generating ECG coherence, or at least generating genuine unconditional love, will be able to resonate with the DNA. Some healers could influence the DNA, although they could not intentionally direct it to either wind or unwind. Other healers could influence the DNA on certain days and not others. Also some healers could bring about conformational changes that would last up to two hours whereas with others the changes would only last 15 minutes. There were also differences in the conformational changes. Normally winding and unwinding of DNA is measured by changes in the absorption of UV light which occur only at the wavelength of $260 \mathrm{~nm}$, but anomalous changes at other wavelengths, notably $310 \mathrm{~nm}$, also occurred.[11] It is quite clear that here, as with all psi experiments, the researchers have to content themselves with getting more successes than failures. Rein's basic thesis that focused human intentionality can produce significant changes in DNA conformation was published in the peer reviewed Acupuncture \& Electro-Therapeutics Research.[11]

In his paper Rein also described the effect of intentionality on the electrical properties of DNA. This was done by measuring the flow of current between two gold electrodes forming an anode and a cathode. The DNA was in aqueous solution $(0.5 \% \mathrm{NaCl}$ or deionized water) and measurements were made of the conductivity of the solution with the DNA present. It was reasoned that the DNA would modulate the conductivity as ions bound to the exterior or interior of the helix would be released and move towards the electrodes. Depending on the charge of the liberated ions they will either enhance or inhibit the flow of the current imposed on the system from the cathode. The healer directed his consciousness towards the DNA while forming different intentions, while three of the experimenters focused on their heart and sent love to the DNA. It was found that although the electrical response of DNA to conscious intention is complex, it is specific.

These responses indicated a dynamic system which was constantly changing, sometimes showing positive spikes and other times showing negative spikes. The direction and amplitude of the spikes can be accounted for by the presence of positive or negative ions appearing at the anode. Overall it was found that some anomalous electrical behavior of DNA is being measured in response to human intentions because the different shapes of the spikes could not be readily explained by traditional electrochemistry. The results also suggest that several different intentions would be more efficacious in bringing about the healing of a particular system. For example in the case of diabetes one might direct the DNA to synthesize more insulin and direct the cell membrane to modify glucose uptake. We shall see later in this paper just how true recent discoveries have confirmed Rein's predictions in this regard.

These experiments were done at HMI in collaboration with Cleve Backster using the methodology he originally used in measuring the energetic communication between plants [12] and which was later applied to measure long distance communication between astronauts and their own leukocytes (cells obtained from the mouth cavity), which is known as the Backster effect.[13] Therefore this methodology is known to detect subtle energies (beyond conventional EM fields) which travel long distances. The type and magnitude of the electrical signals observed from DNA were similar to those Backster previously obtained from plants, bacteria and freshly isolated human leukocytes and were also similar to the signals obtained in these experiments from human skin cells grown in tissue culture. Rein makes the prophetic observation that it is possible that the energetic communication between humans and plants, the Backster effect and between humans and their own cells may be mediated by DNA.

In his discussion Rein referred to a variety of experimental evidence about electrical activity in the DNA.[14-18] Specifically Rein made mention of the pioneering work of Fritz-Albert Popp who initially developed a theory about coherent bio-fields in living tissue [19-20] and went on to discover that the DNA absorbs and emits coherent biophotons which generate these bio-fields [21], and thus initiated a growing body of research known as Biophotonics or Bioinformatics which investigates the effects of these biophotons on natural healing processes as well as identifying potential carcinogens. This research by Popp and his followers clearly demonstrates the existence of EM fields and activity in the DNA as well as in the cells of all animals and plants, but these biophotons being emitted by the DNA are in the frequency range about $10^{15} \mathrm{~Hz}$ (around the middle of the EMR spectrum) whereas it will be revealed below that Rein's experiments about the effect of human intentionality on DNA conformation may be explained by Alpha and $\mathrm{Mu}$ brainwaves which at $7 \mathrm{~Hz}$ or above are at the very bottom of the EMR spectrum.

Rein also referred to recent theories in quantum physics based on a unique topology composed of a catenoid inside the hole of a toroid which was John D. Wheeler's theory to describe how wormholes act as transducers for higher dimensional energy to influx into our 4D space/time reality [22] and he suggested that the DNA had this type of topology as well. Rein proposed the Toroid Antennae Model of DNA function. The toroidal shape of DNA functions as an antennae to allow DNA to sense subtle energies in the environment. In addition the hypothesis states that toroidal DNA acts as a transducer converting subtle energy into conventional EM energy which is then radiated from the DNA to produce a variety of intracellular events at the biochemical level.

In his Toroid Antennae Model of DNA function Rein also made mention of the at that time recent experimental evidence indicating DNA emits EM fields in the form of coherent photons and unpublished research with DNA at the Russian Academy of Science. The latter experiments used a sophisticated machine (Laser correlation spectroscopy) to 
measure the scattering of laser light after it passes through the DNA molecule. The scattered light thereby creates a pattern which is distinctly different from the control in the absence of DNA. However, after removing the DNA from the machine, a third pattern was obtained when the laser was directed at the space where the DNA had been. This experiment elegantly demonstrates for the first time that there is some kind of energy template which is left behind in the machine (after the DNA had been removed) which scattered light in a similar, but different, way as does the physical DNA molecule. This phenomenon was given the name DNA Phantom Effect.

\section{Recent Research Corroborating DNA Psi Effects}

Dr. Glen Rein has had a long association with the Society for Scientific Exploration. I have not been able to find any articles by him in the Journal of Scientific Exploration, however he presented a paper at the 1994 Annual Meeting of the Society for Scientific Exploration entitled "Structural changes in water \& DNA associated with new physiologically measurable states" [23] where he reports that the specific intentions of his subjects were able to change the molecular structure of water and then when this "treated" water was placed into an aqueous solution of placental DNA there were statistically significant changes in the conformation of the DNA as compared with controls.

Rein's observations have been confirmed by two recent papers, one published in the Journal Other Quantitative Biology [24] and the other published in Nature Communications.[25] In 2010 a group of researchers which included Nobel Laureate Dr. Luc Montagnier wrote a paper entitled "DNA waves and water" which corroborates Rein's finding but this time the DNA is changing the molecular structure of the water. Essentially the research found that DNA emits an ultra-low frequency electromagnetic signal that can be stored and transmitted in pure water, and then if you introduce the ingredients necessary for synthesizing the DNA by the polymerase chain reaction - nucleotides, primers, polymerase - to the tube with the pure water that had gained the EM signal then the original DNA sequence would be recreated. Most of the discussion in the paper centers around how the nanostructures in the water can retain the information from the DNA in the form of memory but the essential point is the fact that the information about the original sequence of the DNA was emitted by the DNA as an electromagnetic field. The fact that it is precisely pure water that has this capacity to transmit the signal from the DNA and to retain the integrity of the signal in memory will not come as a surprise when it is remembered that about $65 \%$ of the composition of our bodies is water. In other words it is reasonable to assume that this is a normal metabolic process that is occurring naturally in all creatures containing DNA, including plants. In these experiments a copper solenoid is placed around the tubes and they were exposed to a low intensity electric current oscillating at $7 \mathrm{~Hz}$ produced by an external generator. The magnetic field produced by the external generator is maintained for 18 hours at room temperature. This ambient externally applied magnetic field operating at a minimum of $7 \mathrm{~Hz}$ was found to be essential for the EMS transmission in the water tube; no upper limit was specified. The paper concludes with the observation: "DNA signaling is stimulated by $7 \mathrm{~Hz}$ naturally occurring waves on earth. Waves produced by the human brain are also in the range of $7 \mathrm{~Hz}$. These are interesting questions to be asked and possibly answered".

A recent paper in Nature Communications provides an answer to the second of these questions. The article is entitled "Mind-controlled transgene expression by a wireless-powered optogenetic designer cell implant". The Leader of this research team was a Dr. Martin Fussenegger, a bioengineer at ETH Zurich in Basel, Switzerland. So what did Dr. Fussenegger and his team actually do. He himself states, "We wanted to be able to use brainwaves to control genes. It's the first time anyone has linked synthetic biology and the mind".[26] You will see from the title of this research paper they made use of the comparatively new field called optogenetics. Neuroscientists have now discovered that they can literally, with the flick of a switch, turn the neurons on and off in our brain using light. As well as this they are finding more and more genes that are responsive to light, that is to say EMR.

The Fussenegger team implanted some human kidney cells under the skin of a mouse. In these human cells they had also inserted a gene that was responsive to infrared light. When this gene is activated it causes a cascade of chemical reactions that will lead to the activation of another gene which we will call their target gene. This is the actual gene they wanted to switch on. Also in the mouse alongside the implanted cells they put an infrared LED that could be controlled wirelessly.

They then went about getting some characteristic brainwaves from some human volunteers. The volunteers were taught to produce a "relaxed" pattern of brainwaves from meditation techniques, and they played computer games to produce "deep concentration" brainwaves, and they were taught a technique known as "biofeedback" where they learnt to control their thoughts in such a way as to be able to switch on a set of lights on a computer. These human volunteers were wearing EEG devices that were linked wirelessly to the LED implanted in the mouse, and when that was switched on by any one of these three mental states, it activated the light-responsive gene which in turn started the gene cascade and led to the activation of their target gene; hence their claim that human brainwaves can control genes.

The implant in the mouse was encased in a semi-permeable membrane that allowed nutrients from the animal's blood supply to reach the cells inside and also allowed proteins produced in the implant to pass into the bloodstream. When the target gene was activated it produced 
a human protein that passed into the rodent's bloodstream. Dr. Fussenegger says: "We picked a protein that made an enzyme that was easy to identify in the mouse as proof of concept, but essentially we think we could switch on any target gene we liked".[26]

We have seen from the first article cited that the DNA is stimulated by $\cong 7 \mathrm{~Hz}$ naturally occurring waves on earth, the so-called Schumann resonance. Waves produced by the human brain are also in the range of $7 \mathrm{~Hz}$ and we know from optogenetics that EMR can trigger action potentials in neurons and EMR can turn on light sensitive genes. What all this seems to indicate is that there is some sort of three way communications or biofeedback going on between the DNA, the neurons of the brain, and the Earth's Schumann resonance. If this is in fact the case you have not only a complete explanation for psi or psychic phenomena in humans, but you also have an explanation for other sorts of unexplained phenomena in nature such as the way termites which are actually blind can act in concert to build their nests, how various bird species can literally navigate to the other end of the earth in order to breed and then return to their normal habitat, how schools of fish and flocks of birds can change direction in apparent unison, and indeed how plants can communicate with each other. All of these other types of phenomena are very well documented but essentially remain a scientific mystery. What we humans call psi phenomena is simply the way the lower animals and the plant kingdom communicate with each other as a matter of course. It is psi phenomena to us for the simple fact that it is subconscious and remains subtle and inexplicable to us because we have the much more tangible and conscious means of communication, namely language.

\section{The Schumann Resonance and Brainwaves}

In fact it has been known for a long time that there is a connection between brainwaves, the Schumann resonance and psi. An article by Iona Miller published in July 2013 in the Journal of Consciousness Exploration \& Research entitled "Schumann Resonance, Psychophysical Regulation \& Psi" sets out comprehensively the connection between Schumann resonance and brainwaves [27]. This article is incomplete only for the fact that it does not also make the connection with the EMR being emitted from the DNA. Although Iona Miller does actually say and I quote "All biological processes are a function of electromagnetic field interactions. EM fields are the connecting link between the world of form and resonant patterns. They store gestalts or patterns of information. The bridge connecting solar system resonances and brain frequencies resides in our human DNA helix, which co-evolved in the Earth's environment".

The Schumann resonance (SR) is defined as a set of resonant modes or spectrum peaks, between 7.83 and $45 \mathrm{~Hz}$, in the extremely low frequency (ELF) portion of the Earth's electromagnetic field spectrum. The fundamental Schumann Resonance is a standing wave in the atmosphere around $8 \mathrm{~Hz}$. Human brainwaves are entrained to this pulse emitting theta and alpha frequencies in the same EMF region. The reciprocal system functions as a phase-locked loop. Basically a phase-locked loop is a control system that generates an output signal whose phase is related to the phase of an input "reference" signal. This is precisely what was described in the paper on DNA waves and water mentioned earlier.

The Schumann resonance is necessary for mammalian growth and repair; such signals in guide waves are the meta-drivers of biological processes, homeostasis and adaptation. We cannot thrive without them. Cells respond between $3-25 \mathrm{~Hz}$. Frequencies outside this range have little or no effect.

In the early days of the space race it was found that cosmonauts that were outside the earth's magnetic field for extended periods lost $80 \%$ of their bone density. Consequently Schumann wave generators $(7.83 \mathrm{~Hz})$ were developed for space flights which overcame this side effect. [28]

We are bathed in a sea of natural low-frequency electromagnetic (EM) fields from conception to death. The brain is an electromagnetic system synchronized by the Schumann Resonance signal that continuously stabilizes the brain wave activity. [29]

Research has shown that the Schumann resonances can modulate human health indicators such as blood pressure, cardiac and neurological disease, reaction time, neuroendocrine sensitivities. [30-33]

Iona Miller refers to the research of Robert C. Beck. He found that the human body has numerous very specific frequencies that trigger production of different endorphins, beta-endorphins, catecholamines, enkephalins, dynorphins, proteins, and stem cells. Specifically he found by measuring the brainwave activity in "healers" from many different traditions (including psychics, shamans, faith healers, practitioners of Wicca and an Hawaiian kahuna) that when they are actually performing a healing their EEG recordings were on average around 7.8-8.0 cycles/second, that is to say the Schumann frequency.[34] This would appear to be direct evidence that the psi activity reported by Rein on conscious intention on the DNA can be explained by the healers brainwaves being phase and frequency synchronized with the Schumann residence.

Raw EEG frequency bands include gamma $(25-60 \mathrm{~Hz})$; beta $(12-25 \mathrm{~Hz})$; alpha $(7-12 \mathrm{~Hz})$; theta $(4-7 \mathrm{~Hz})$; and delta (less than $4 \mathrm{~Hz}$ ). Their ranges overlap one another along the frequency spectrum by $0.5 \mathrm{~Hz}$ or more. In other words the brainwaves are being generated at multiple frequencies and if it is true that brainwaves are actually modulating the synthesis and activity of various neurotransmitters, proteins and hormones within the body then different bodily functions and organs as well as the DNA itself must respond to a single frequency only and can then tune into the brainwaves similar to the way we tune in to our favorite radio 
station.

Iona Miller tells us that alpha waves are present during dreaming and light meditation when the eyes are closed. Alpha-wave biofeedback is considered a consciousness self-regulation technique. This induces a state of deep relaxation, but not quite meditation. In alpha, we begin to access the wealth of creativity that lies just below our conscious awareness. When the goal is alpha, either in meditation or in biofeedback, it means entraining with the primary SR. As a matter of commonsense it is plain that this is the zone of consciousness that the healers are likely to be accessing in order to perform the psi acts on the DNA previously described. They are not likely to be able to do those acts in deep sleep or deep meditation for instance, nor in the state of alert consciousness when the brainwave frequencies increase considerably.

Iona Miller also refers to a wide variety of theorists who in one way or another have hypothesized that EM fields outside the body are crucial for our consciousness, in particular Lian Sidorov who edited the Journal of Non-Locality and Remote Mental Interactions (JNLRMI). [35-38] In 1973, Miller, Webb and Dickson described DNA as a holographic projector.[39] In other words, genes encode and express themselves via light and radio waves, or acoustical holography. This theory would appear to directly anticipate the findings of Dr. Luc Montaigner mentioned earlier that the waves coming from the DNA actually contain information about its own base sequence. To describe these DNA waves as a holographic projection of the master molecule, the DNA itself, would seem to be most apropos. Also the theory of Beal in 1996 that liquid crystals (DNA, brain ventricles, and cellular structures) in the human body may operate as antennae for detecting and decoding such global and local ELF signals.[40] This suggestion also seems to anticipate the findings of Dr. Luc Montaigner that the holographic projection of the DNA is stimulated and modulated by a background EMR set at a similar frequency to the Schumann resonance.

Iona Miller also referred to the landmark experiments of Robert Becker in the mid-1980s which led him to the conclusion that subtle current of EMR in the body could reach far deeper into our genetic and consciousness control mechanisms. [41] It is suggested that the findings of Robert Becker have anticipated the research of Dr. Fussenegger mentioned above where it was found that brainwaves can actually control gene expression. In this recent experiment he used a standard commercial low-cost Brain-Computer Interface (BCI) headset to record the brain waves of the human subjects. It is stated in his paper that this headset recorded all the normal brain waves, the delta, theta, low alpha, high alpha, beta and gamma bands. However in his paper all this brainwave activity was fed into a meditation meter and it is not specified exactly what brainwave frequencies actually resulted in the expression of specific genes. However I can report that in this area, generally known as cybernetics, which advances the design of man-machine interfaces as well as Brain-Computer
Interfaces there is only one primary band of frequency that is almost invariably utilized and that is the alpha band brainwaves. See for instance the paper in the journal IEICE Transactions "The Development of BCI Using Alpha Waves for Controlling the Robot Arm" for a fairly typical explanation of this process. [42]

It just so happens there is another band, the so-called mu rhythm, which actually operates within the identical frequency window as the alpha waves.[43] This mu rhythm extends across the motor cortex at the back of the head from ear to ear, and it is the suppression of this mu rhythm that is also used widely in BCIs. Let me repeat that this mu rhythm is operating at the same frequency as the alpha rhythm, as well as the Schumann resonance. A person suppresses mu wave patterns when he or she performs a motor action or, with practice, when he or she visualizes performing a motor action. This suppression is called desynchronization of the wave because EEG wave forms are caused by large numbers of neurons firing in synchrony.

In fact the mu wave is even suppressed when one observes another person performing a motor action or an abstract motion with biological characteristics. This has been known since 2005. This phenomenon has been given a special name, the Mirror Neuron System. See the paper in the journal PLOS Biology "Grasping the Intentions of Others with One's Own Mirror Neuron System”. [44] The importance of this cannot be overstated for psi research. It is now known that the mirror neurons in our own brain fire when we observe someone else performing an act, just as if we were performing the act ourselves. And the brainwaves being generated are in the same frequency range as the Schumann resonance as well as the background resonance for the DNA EMR. This phenomenon can therefore explain any amount of psychic happenings that have been reported over the centuries.

To give you a concrete example, who better than Aleister Crowley aka The Beast or "the wickedest man in the world".[45] To demonstrate his powers Crowley is said to have fallen in-step behind a banker who was walking along Fifth Avenue in New York. He walked behind him imitating his walk and concentrated on the back of his head where his motor cortex is. Then Crowley suddenly buckled at the knees, squatted for a split second on his haunches, then shot up again. The banker in mid-stride buckled and collapsed to the sidewalk, and as he was getting up he was looking around for the banana skin with a most puzzled look. It is suggested that Crowley was able to synchronize his mu rhythm with that of the banker and then by feigning a fall himself he desynchronized the mu rhythm in the motor cortex of the banker and caused him to tumble in fact. Crowley knew how to activate the mirror neurons in the banker's brain, a scientific explanation for his "magic" powers.

\section{Conclusions}

I have entitled this paper a review of psi activity in the 
DNA but as you have seen I have only been able to give a very brief account of all the material that is available specifically dealing with phenomena relating to mental communication between human beings and the organic molecule called the DNA. The research done during the 1980s and 1990s principally at the HeartMath Institute in Boulder Creek, CA has been almost universally ignored or dismissed as paranormal pseudo-science. The fact is however that EMR in the DNA is well and truly mainstream science. There is simply a vast amount of research data in the mainstream literature about EMR in the DNA. A fairly typical summary of EM activity in the DNA can be found in "Electric oscillation and coupling of chromatin regulate chromosome packaging and transcription in eukaryotic cells".[46] Please note specifically in that article all the data on EMR in the so-called "junk DNA", that is to say it has nothing whatsoever to do with the $3 \%$ or so of the DNA that comprises our chemical genes. This is not just the DNA of animals that is emitting EMR but also the DNA of plants. Mainstream biologists studying communication in plants have recorded a prodigious amount of EM activity specifically in the roots of plants. In fact there exists now a society of biologists called the Society for Plant Neurobiology, who have revived the original idea of the great Charles Darwin himself that the primary root in the plant acts as an embryonic brain.[47] Plants of course do not have neurons, but they do have DNA that emits EMR. In addition in the past ten years or so a whole new area of genetics has exploded on the scene, called optogenetics. The body of data about genes that are responsive to EMR is growing exponentially as is the data that action potentials in neurons can be triggered by EMR from the DNA; that is to say there is undoubtedly a two way communication between the neurons of the brain and the DNA precisely by means of EMR aka brainwaves. The point of this paper is therefore that those somewhat surreal psi experiments conducted in the latter decades of the $20^{\text {th }}$ century at the HeartMath Institute are pseudoscience no longer.

\section{REFERENCES}

[1] Rein, G. Effect of conscious intention on human DNA, Proceeds of the International Forum on New Science, Denver CO., October, 1996.

[2] Jahn, R.G., \& Dunne, B.J. On the quantum mechanics of consciousness, with applications to anomalous phenomena, Found. Phys. 16: 721-36, 1986.

[3] Braud, W. On the use of living target systems in distant mental influence research. In: B. Shapin \& L. Coly (eds). Psi Research Methodology: A Reexamination, Parapsychology Foundation, N.Y., 1989.

[4] Benor, D. J. Survey of spiritual healing research, Complementary Med. Res. 4: 9-33, 1990.
[5] Rauscher, E.A., \& Rubik, B.A. Human volitional effects on a model bacterial system, Psi. Res. 2, 38-47, 1983.

[6] Sweet. Full citation is not available, 1991.

[7] Rein, G. Healing with Love: A Breakthrough Mind/Body Medical Program for Healing Yourself and Others, Harper, San Francisco, 1992.

[8] Rein, G., \& McCraty, R. Modulation of DNA by coherent heart frequencies, Proc. Third Internat. Soc. Study Subtle Energy \& Med. Conf., Monterey, CA., 1993.

[9] Rein, G., \& McCraty R. Local and Non-local Effects of Coherent Heart Frequencies on Conformational Changes in DNA, Proc. Joint USPA/IAPR Psychotronics Conf., Milwaukee, WI., 1993.

[10] Rein, G., \& McCraty, R. DNA As a Detector of Subtle Energies, Proc. Fourth Internat. Soc. Study Subtle Energy \& Med. Conf., Monterey, CA., 1994.

[11] Rein, G. The In Vitro Effect of Bioenergy on the Conformational States of Human DNA in Aqueous Solutions, Acupuncture and Electrotherapeutics Res. 20: 173-180, 1995.

[12] Backster, C. Evidence of a primary perception in plant life, Internat. J. Parapsychol. 10: 329-348, 1968.

[13] Backster, C., \& White, S. G. Bio-communications capability: human donors and in vitro Leukocytes, Internat. J. Biosocial Res. 7: 132-146, 1985.

[14] Bjorgum, O., \& Godal, T. On Beltrami vector fields and flows (Pt.2), Universitet I Bergen Arbok, 1952.

[15] Olariu, S., \& Popescu, I. The Quantum Effect of Electromagnetic Fluxes, Rev. Modern Phys, 57: 339-348, 1985.

[16] Beltrami, E. Notes on the mathematical theory of electrodynamic solenoids, Int. J. Fusion Energy 3: 43-50, 1985.

[17] Beltrami, E. Considerazioni idrodinamiche, Rend. Inst. Lombardo Acad. Sci. Lett, 22: 121-131, 1989.

[18] Poponin, V. The DNA phantom effect: direct measurement of a new field in the vacuum substructure, Ann. Conf. on Treatment and Res. Experienced Anomalous Trauma, San Rafael, CA., 1995.

[19] Popp, F-A., \& Ruth, B. (eds.) Electromagnetic Bioinformation, Urban \& Schwarzenburg, Baltimore, 1979.

[20] Popp, F-A., \& Ruth, B. Emission of Visible and Ultraviolet Radiation by Active Biological Systems, Collective Phenom. 3: 187-214, 1981.

[21] Rattemeyer, M., Popp, F. A., \& Nagl, W. Evidence of photon emission from DNA in living systems, Naturwissen 68: $572-580,1981$.

[22] Wheeler, J.A. Geometrodynamics, Academic Press, NY., 1962.

[23] Rein, G., \& McCraty R. Structural Changes in Water \& DNA Associated with New Physiologically Measurable States, Journal of Scientific Exploration, 8(3): 438-439, 1994.

[24] Montagnier, L., Aissa, J., Del Giudice, E., Lavallee, C., Tedeschi, A., \& Vitiello, G. DNA waves and water, Journal 
of Other Quantitative Biology, 1012.5166v1, 2010.

[25] Fussenegger, M., Folcher, M., Oesterle, S., Zwicky, K., Thekkottil, T., Heymoz, J., Hohmann, M., Christen, M., Daoud El-Baba, M., \& Peter Buchmann, P. Mind-controlled transgene expression by a wireless-powered optogenetic designer cell implant, Nature Communications, 5: 5392, 2015.

[26] Thomson, H. Human thoughts used to switch on genes, New Scientist 11/15/2014.

[27] Miller, I. Schumann Resonance, Psychophysical Regulation \& Psi, Journal of Consciousness Exploration \& Research 4:6:599-612, 2013.

[28] Wilkes, E. Nature's Secret Messages, Hay House, 2010.

[29] Blackman, C.F. ELF effects on calcium homeostasis. In: BW Wilson, RG Stevens, LE Anderson (Eds). Extremely low frequency electromagnetic fields: The question of cancer, Publ. Battelle Press Columbus, 187-208, 1990.

[30] Roffey, L. The Bioelectronic Basis for "Healing Energies: Charge and Field Effects as a Basis for Complementary Medical Techniques, DNA Decipher Journal, 2, 2012.

[31] Ludwig, H.W. Electromagnetic multiresonance - the basis of homeopathy and biophysical therapy, Proceedings 42nd Congress Int. Homeopathic Med League, Am. Institute Homeopathy, Washington, D.C., 1987.

[32] Bentov, I. Stalking the Wild Pendulum. In. E.P. Dutton (ed) On the Mechanics of Consciousness, Destiny Books, 1988.

[33] Hainsworth, L.B. The Effect of Geophysical Phenomena on Human Health, Speculations in Science and Technology, Vol. 6, No. 5., 1983.

[34] Oschman, J. Energy Medicine, Churchill Livingstone, 2000.

[35] Sidorov, L. On the possible mechanism of intent in paranormal phenomena, Journal of Theoretics, 2001.

[36] Sidorov, L. Control systems, transduction arrays and psi healing: an experimental basis for human potential science, JNLRMI, Vol. I No.2., 2002.
[37] Sidorov, L. The imprinting and transmission of mentally-directed bioinformation, JNLRMI, Vol. 1 No. 1, Jan. 2002.

[38] Sidorov, L. The tip of the iceberg: placebo, experimenter expectation and interference phenomena in subconscious information flow, Journal of Nonlocality, Vol. II, No.1., 2013.

[39] Miller, R.A., Webb, B, \& Dickson, D. Holographic Concept of Reality and Embryonic Holography, Presented at the Omniversal Symposium, California State College at Sonoma, 1973.

[40] Beal, J.B. Biosystems liquid crystals and potential effects of natural and artificial electromagnetic fields (EMFs), Second Annual Advanced Water Sciences Symposium, Exploratory Session, Dallas, TX., 1996.

[41] Becker, R. Electromagnetism and Life, State University of New York Press, 1985.

[42] Inoue, S., Akiyama, Y., Izumi, Y., \& Nishuima, S. The Development of BCI Using Alpha Waves for Controlling the Robot Arm, IEICE Transactions on Communications Vol.E91-B No.7, 2125-2132, 2008.

[43] Hundia, R. (2015). Brain Computer Interface-Controlling Devices Utilizing The Alpha Brain Waves, International Journal of Scientific \& Technology Research Vol. 4, Issue 01, 2015.

[44] Iacoboni, M., Molnar-Szakacs, I., Gallese, V., Buccino, G., Mazziotta, J.C., \& Rizzolati, G. Grasping the Intentions of Others with One's Own Mirror Neuron System, PLoS Biol 3(3): e79, 2005.

[45] Wilson, C. (1971). The Occult, Panther Granada, London, 1971.

[46] Zhao, Y., \& Zhan, Q. Electric oscillation and coupling of chromatin regulate chromosome packaging and transcription in eukaryotic cells, Theoretical Biology and Medical Modelling 9:27, 2012.

[47] Darwin, C. The Power of Movement in Plants, Public Domain Book, 1880. 